\title{
Validation and refinement of the clinical staging model in a French cohort of outpatient with schizophrenia (FACE-SZ).
}

\section{Running title: Clinical staging in schizophrenia}

Godin Ophelia $^{\mathrm{a}, \mathrm{b}}$, PhD, Fond Guillaume ${ }^{\mathrm{aq}}$, MD, PhD, Bulzacka Ewa ${ }^{\mathrm{a}, \mathrm{p}}$, Msc, Schürhoff Frank ${ }^{\mathrm{a}, \mathrm{p}}, \mathrm{MD}$ PhD, Boyer Laurent $^{\mathrm{a}, \mathrm{c}}, \mathrm{MD} \mathrm{PhD}$, Andre $\mathrm{M}^{\mathrm{a}, \mathrm{f}}, \mathrm{PhD}$, Andrianarisoa Meja ${ }^{\mathrm{a}, \mathrm{p}}, \mathrm{MD} \mathrm{PhD}$, Aouizerate Bruno ${ }^{\mathrm{a}, \mathrm{d}, \mathrm{n}}, \mathrm{MD} \mathrm{PhD}$, Berna Fabrice $^{\mathrm{a}, \mathrm{e}}, \mathrm{MD} \mathrm{PhD}$, Capdevielle Delphine ${ }^{\mathrm{a}, \mathrm{f}}, \mathrm{MD} \mathrm{PhD}$, Chereau Isabelle ${ }^{\mathrm{a}, \mathrm{g}}, \mathrm{MD}$, Dorey Jean-Michel ${ }^{\mathrm{a}, \mathrm{h}}$, Msc, Dubertret Caroline ${ }^{\mathrm{a}, \mathrm{i}}$, MD PhD, Dubreucq Julien ${ }^{\mathrm{a}, \mathrm{j}}, \mathrm{MD}$, Faget Catherine ${ }^{\mathrm{a}, \mathrm{k}}$, MD, Lancon Christophe ${ }^{\mathrm{a}, \mathrm{k}}$, MD PhD, Leignier Sylvain ${ }^{\mathrm{a}, \mathrm{j}}, \mathrm{MSc}$, Mallet Jasmina ${ }^{\mathrm{a}, \mathrm{i}}, \mathrm{MD} \mathrm{PhD}^{\mathrm{a}, \mathrm{i}}$, Misdrahi David ${ }^{\mathrm{a}, \mathrm{d}, \mathrm{o}}, \mathrm{MD}$, Passerieux Christine ${ }^{\mathrm{a}, \mathrm{l}}$, MD PhD, Rey Romain ${ }^{\mathrm{a}, \mathrm{h}}, \mathrm{MD}$, Roux Paul ${ }^{\mathrm{a}, \mathrm{l}}, \mathrm{MD} \mathrm{PhD}$, Vidailhet Pierre ${ }^{\mathrm{a}, \mathrm{e}}, \mathrm{MD} \mathrm{PhD}$, Costagliola Dominique ${ }^{\mathrm{b}}, \mathrm{PhD}$, Leboyer Marion ${ }^{\mathrm{a}, \mathrm{p}}, \mathrm{MD}, \mathrm{PhD}$, Llorca Pierre-Michel ${ }^{\mathrm{a}, \mathrm{g}}, \mathrm{MD} \mathrm{PhD}$

And for the FACE-SZ (FondaMental Academic Centers of Expertise for Schizophrenia) group*

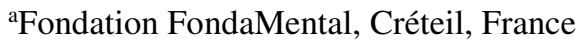

bSorbonne Universités, UPMC Univ Paris 06, UMR_S 1136, Institut Pierre Louis d'Epidémiologie et de Santé Publique; INSERM, UMR_S 1136, Institut Pierre Louis d'Epidémiologie et de Santé Publique, F75013, Paris, France

'Pôle Psychiatrie Universitaire, CHU Sainte-Marguerite, F-13274 Marseille cedex 09, France

${ }^{\mathrm{d}}$ Centre Hospitalier Charles Perrens, Université de Bordeaux, F-33076 Bordeaux, France

eHôpitaux Universitaires de Strasbourg, Université de Strasbourg, INSERM U1114, Fédération de Médecine Translationnelle de Strasbourg, Strasbourg, France

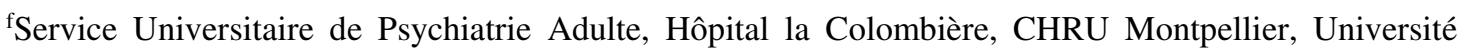
Montpellier 1, Inserm 1061, Montpellier, France.

gCMP B, CHU, Université d'Auvergne, BP 6963003 Clermont-Ferrand Cedex 1, France.

hINSERM U1028, CNRS UMR5292, Université Claude Bernard Lyon 1, Centre de Recherche en Neurosciences de Lyon, Equipe PSYR2, Centre Hospitalier Le Vinatier, Pole Est, 95 bd Pinel, BP 30039, 69678 Bron Cedex, France

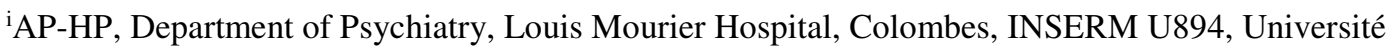
Paris Diderot, Sorbonne Paris Cité, Faculté de Médecine, France.

jCentre Référent de Réhabilitation Psychosociale, $\mathrm{CH}$ Alpes Isère, Grenoble, France.

${ }^{\text {k} A s s i s t a n c e ~ P u b l i q u e ~ d e s ~ H o ̂ p i t a u x ~ d e ~ M a r s e i l l e ~(A P-H M), ~ P o ̂ l e ~ U n i v e r s i t a i r e ~ d e ~ P s y c h i a t r i e, ~ M a r s e i l l e, ~}$ France.

${ }^{1}$ Service Universitaire de Psychiatrie d'Adultes, Centre Hospitalier de Versailles, UFR des Sciences de la Santé Simone Veil, Université Versailles Saint-Quentin-en-Yvelines, Versailles, France 
mPellegrin University Hospital, Bordeaux University, USR CNRS 3413 SANPSY, Research Unit, 33000 Bordeaux, France.

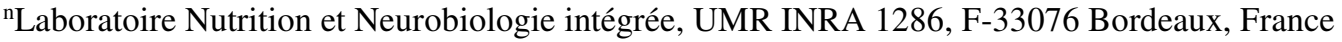

${ }^{\circ}$ CNRS UMR 5287-INCIA

PINSERM U955, Translational Psychiatry laboratory, Mondor Institute of Biomedical Research, Université Paris Est Creteil, AP-HP, DHU Pe-PSY, Pôle de Psychiatrie des Hôpitaux Universitaires H Mondor, Créteil, France

qAP-HM, Aix-Marseille Univ, Faculté de Médecine - Secteur Timone, EA 3279: CEReSS, 13005 Marseille, France

\section{*FACE-SZ Group}

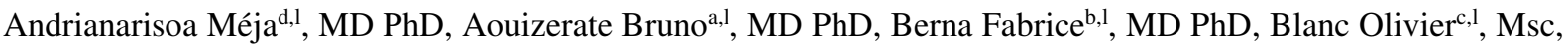
Brunel Lore $^{\mathrm{d}, 1}$, Msc, Bulzacka Ewa ${ }^{\mathrm{d}, 1}$, Msc, Capdevielle Delphine ${ }^{\mathrm{e}, 1}$, MD PhD, Chéreau-Boudet Isabelle ${ }^{\mathrm{c}, 1}$, MD, Chesnoy-Servanin Gabrielle ${ }^{\mathrm{f}, 1}$, Msc, Danion Jean-Marie ${ }^{\mathrm{b}, 1}$, MD, D’Amato Thierry ${ }^{\mathrm{f}, 1}$, MD PhD, Deloge Arnaud ${ }^{\mathrm{g}, 1}$, MD PhD, Delorme Claire ${ }^{\mathrm{h}, \mathrm{l}}$, Msc, Denizot Hélène ${ }^{\mathrm{c}, \mathrm{l}}$, MD, Dorey Jean-Michel ${ }^{\mathrm{f}, \mathrm{l}}$, MD, Dubertret Caroline ${ }^{\mathrm{i}, \mathrm{l}}$, MD PhD, Dubreucq Julien ${ }^{\mathrm{h}, \mathrm{l}}$, MD, Faget Catherine ${ }^{\mathrm{j}, \mathrm{l}}$, MD, Fluttaz Cécile ${ }^{\mathrm{h}, \mathrm{l}}$, Msc, Fond Guillaume ${ }^{\mathrm{d}, \mathrm{l}}$, MD, Fonteneau Sandrine $^{\mathrm{k}, \mathrm{l}}$, Msc, Gabayet Franck ${ }^{\mathrm{h}, \mathrm{l}}$, Msc, Giraud-Baro Elisabeth ${ }^{\mathrm{h}, \mathrm{l}}$, MD, Hardy-Baylé Marie-Christine ${ }^{\mathrm{k}, \mathrm{l}}$, MD PhD, Lacelle Delphine ${ }^{\mathrm{c}, 1}$, Msc, Lançon Christophe ${ }^{\mathrm{j}, 1}$, MD PhD, Laouamri Hakim¹, Msc, Leboyer Marion ${ }^{\mathrm{d}, 1}$, MD PhD, Le Gloahec Tifenn ${ }^{\mathrm{d}, l}$, Msc, Le Strat Yann ${ }^{\mathrm{i}, 1}$, MD PhD, Llorca ${ }^{\mathrm{c}, 1}$ Pierre-Michel, MD PhD, Mallet Jasmina ${ }^{\mathrm{i}, \mathrm{j}}$, MD PhD, Metairie Emeline $\mathrm{j}^{\mathrm{j}, 1}$, Msc, Misdrahi David ${ }^{\mathrm{g}, 1}$, MD PhD, Passerieux Christine ${ }^{\mathrm{k}, 1}$, MD PhD, Peri Pauline ${ }^{\mathrm{j}, 1}$, Msc, Pires Sylvie ${ }^{\mathrm{c}, \mathrm{l}}$, Msc, Portalier Céline ${ }^{\mathrm{i}, 1}$, Msc, Rey Romain ${ }^{\mathrm{f}, 1}$, MD, Roman Céline ${ }^{\mathrm{h}, \mathrm{l}}$, Msc, Sebilleau Mathilde $^{\mathrm{k}, 1}$, Msc, Schandrin Aurélie ${ }^{\mathrm{e}, \mathrm{l}}$, MD, Schneider Priscille ${ }^{\mathrm{b}, \mathrm{l}}$, Schurhoff Franck ${ }^{\mathrm{d}, 1}$, MD PhD, Tessier Arnaud $^{\mathrm{g}, 1}$, Msc, Tronche Anne-Marie ${ }^{\mathrm{c}, 1}$, MD, Urbach Mathieu ${ }^{\mathrm{k}, 1}$, MD, Vaillant Florence ${ }^{\mathrm{j}, 1}$, Msc, Vehier Aurélie ${ }^{\mathrm{f}, 1}$, Msc, Vidailhet Pierre ${ }^{\mathrm{b}, 1}$, MD PhD, Vilà Estelle ${ }^{\mathrm{g}, 1}$, Msc, Yazbek Hanan ${ }^{\mathrm{e}, 1}$, PhD, Zinetti-Bertschy Anna ${ }^{\mathrm{b}, 1}$, Msc.

${ }^{a}$ Centre Hospitalier Charles Perrens, F-33076 Bordeaux, France; Université de Bordeaux, INSERM, Neurocentre Magendie, Physiopathologie de la Plasticité Neuronale, U862, F-33000 Bordeaux, France

bHôpitaux Universitaires de Strasbourg, Université de Strasbourg, INSERM U1114, Fédération de Médecine Translationnelle de Strasbourg, Strasbourg, France

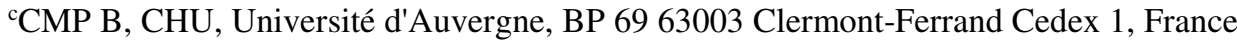

dAP-HP, DHU Pe-PSY, Pôle de Psychiatrie et d'Addictologie des Hôpitaux Universitaires H Mondor,INSERM U955, Eq 15 Psychiatrie Translationelle, Université Paris Est-Créteil, Créteil, France

eService Universitaire de Psychiatrie Adulte, Hôpital la Colombière, CHRU Montpellier, Université Montpellier 1, Inserm 1061, Montpellier, France.

fINSERM U1028, CNRS UMR5292, Université Claude Bernard Lyon 1, Centre de Recherche en Neurosciences de Lyon, Equipe PSYR2, Centre Hospitalier Le Vinatier, Pole Est, 95 bd Pinel, BP 30039, 69678 Bron Cedex, France

${ }^{\text {g}}$ Centre Hospitalier Charles Perrens, F-33076 Bordeaux, France; Université de Bordeaux, CNRS UMR 5287-INCIA, France

${ }^{\text {h}}$ Centre Référent de Réhabilitation Psychosociale, $\mathrm{CH}$ Alpes Isère, Grenoble, France.

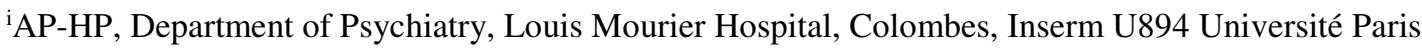
Diderot, Sorbonne Paris Cité, Faculté de médecine, France. 
jAssistance Publique des Hôpitaux de Marseille (AP-HM), Pôle Universitaire de Psychiatrie, Marseille, France.

${ }^{\mathrm{k}}$ Service Universitaire de Psychiatrie d'Adultes, Centre Hospitalier de Versailles, UFR des Sciences de la Santé Simone Veil, Université Versailles Saint-Quentin-en-Yvelines, Versailles, France

${ }^{\text {l} F o n d a t i o n ~ F o n d a M e n t a l, ~ C r e ́ t e i l, ~ F r a n c e ~}$

\section{Corresponding author}

Ophélia Godin

Institut Pierre Louis d'Epidémiologie et de Santé Publique

UMR_S 1136

Hôpital La Salpêtriere,

75651 Paris Cédex 13, France

(Tel: +33 142162546, Fax: +33 142162541, e-mail : opheliagodin@yahoo.com.

Abstract word count: 208 


\begin{abstract}
Objective: Existing staging models have not been fully validated. Thus, after classifying patients with schizophrenia according to the staging model proposed by McGorry et al. (2010), we explored the validity of this staging model and its stability after one-year of follow-up.
\end{abstract}

Method: Using unsupervised machine-learning algorithm, we classified 770 outpatients into 5 clinical stages, the highest being the most severe. Analyses of (co)variance were performed to compare each stage in regard to socio-demographics factors, clinical characteristics, comorbidities, ongoing treatment and neuropsychological profiles.

Results: The precision of clinical staging can be improved by sub-dividing intermediate stages (II and III). Clinical validators of class IV include the presence of concomitant major depressive episode (42.6\% in stage IV versus $3.4 \%$ in stage IIa), more severe cognitive profile, lower adherence to medication and prescription of more than 3 psychotropic medications. Follow-up at one-year showed good stability of each stage.

Conclusion: Clinical staging in schizophrenia could be improved by adding clinical elements such as mood symptoms and cognition to severity, relapses and global functioning. In terms of therapeutic strategies, attention needs to be paid on the factors associated with the more stages of schizophrenia such as treatment of comorbid depression, reduction of the number of concomitant psychotropic medications, improvement of treatment adherence, and prescription of cognitive remediation.

Keyword: Clinical staging, schizophrenia, prognosis, cognition, comorbidity 


\section{Introduction}

Clinical staging has been widely used to predict course and to optimize treatment in most chronic medical disorders, but until recently it has relatively been neglected in psychiatry (1). Clinical staging differs from conventional diagnostic practice in that it defines not only the extent of progression of a disorder at a given point in time, but also where a person currently lies along the illness course continuum (2). The last two decades have seen the gradual emergence of clinical staging in psychiatric disorders. In schizophrenia, staging models are likely to have heuristic utility in the understanding of illness progression, improving the exploration of relationships of stages with biomarkers and psychosocial risk factors. A major advantage of clinical staging models is the guidance they provide to clinicians to estimate prognosis and to define therapeutic strategies relevant for each stage.

Different theoretical staging models, have been proposed such as stage 1 as the "latent stage" to stage IV as the "chronic and refractory stage" $(2,3)$ without necessarily identifying external validators of these different propositions. Few follow-up studies have as of yet been performed and most of them have been done in the field of bipolar disorder (4-6). In schizophrenia, to the best of our knowledge, only three studies have applied clinical staging models in individuals with schizophrenia. In a sample of 171 individuals at a high risk of psychosis followed-up over 3 years, Carrion and colleagues showed that the severity of early prodromal symptoms plays a critical role in determining clinical outcome, including the risk of psychosis, time to emergence and medication treatment (7). However, this study only focused on the early phases of the illness and transition to psychosis. In a sample of 203 patients with schizophrenia, Ortiz et al. investigated whether clinical and psychopathological differences exist between first-episode schizophrenia and multiple-episode patients; however they focused on hospitalized patients (8). Tedja et al., concluded that clinical staging was applicable to schizophrenia (9) by investigating a retrospective cohort of 649 patients 
diagnosed with schizophrenia, followed up for 3 years. However, in order to fulfill the defined staging criteria, only $20 \%$ of the initial sample could be assignable to a clinical stage, thereby limiting the generalization of their results. To date, no study has applied a clinical staging framework, as defined by McGorry et al. (2010) (3), in a large systematically recruited cohort of outpatients with schizophrenia.

\section{Aims of the study}

The present study has three aims: (i) to classify patients with schizophrenia according to the staging model proposed by McGorry et al (2010), using number of episodes, daily functioning and current illness severity, in a prospective cohort of 770 patients (ii) to use clinical, cognitive and treatment characteristics to explore the validity of this staging model, and (iii) to explore the stability of these different stages after one-year of follow-up. 


\section{Materials \& Method}

\section{Design}

The study sample was composed of outpatients, assessed and followed up in a French network of schizophrenia expert centers (7). The FACE-SZ (FondaMental Advanced Centers of Expertise- Schizophrenia) cohort is based on a French network of 10 Schizophrenia Expert Centers, coordinated by the FondaMental Foundation (www.fondation-fondamental.org), Clinically stable outpatients (defined by the absence of hospitalizations or changes in treatment during the eight weeks before inclusion) above the age of 16 years, with a DSM-IVTR diagnosis of schizophrenia (SZ) or schizoaffective disorder, were included in this study.

Diagnosis was confirmed by two trained psychiatrists of the Schizophrenia Expert Centers Network. After initial examination, patients were seen every year at the Expert Center during a 3 years follow-up, using a standardized and systematic assessment. Only one-year follow-up data are available to date.

The study was carried out in accordance with ethical principles for medical research involving humans (WMA, Declaration of Helsinki). The assessment protocol was approved by the relevant ethical review board (CPP-Ile de France IX, January 18, 2010). All data were collected anonymously. A non-opposition form was signed by all participants as this study including data coming from regular healthcare assessments.

\section{Assessment}

\section{Clinical and Sociodemographic Measures}

All patients were interviewed by a psychiatrist using the Structured Clinical Interview for Mental Disorders (SCID 1.0) to confirm diagnosis. Information concerning education, the onset and course of the illness, family history, psychiatric and somatic comorbidities were also recorded. Schizophrenic symptomatology was assessed using the Positive And Negative 
Syndrome Scale (PANSS) (10) and the PANSS five-factor model for the PANSS items in order to evaluate specific domains of symptomatology (11).

Current depressive symptoms were evaluated using the Calgary Depression Rating Scale for Schizophrenia (12) and manic symptoms were assessed using the Young Mania Rating Scale (13). Global social functioning was evaluated using the Global Assessment of Functioning scale (GAF). Ongoing psychotropic treatment, adherence to treatment, evaluated using the French version of the Medication Adherence Rating Scale (MARS) (14), current cannabis use, alcohol consumption, and tobacco smoking were also recorded. Quality of life was assessed using the EQ5D scale. Chlorpromazine equivalent doses (CPZ100eq) were calculated according to the minimum effective dose method (15). Akathisia was measured with the Barnes Akathisia Scale (16) and presence of parkinsonism with the Simpson-Angus scale of extrapyramidal symptoms (17).

\section{Neuropsychological assessment}

Neuropsychological performances were assessed with a comprehensive test battery covering a wide range of relevant aspects of cognition in schizophrenia. The National Adult Reading Test (NART) (18) provides an estimate of premorbid intellectual ability based on current reading performance. Wechsler Adult Intelligence Scale - 3rd Edition (WAIS-III) (19) provides a measure of general intellectual function in older adolescents and adults. California Verbal Learning Test $(C V L T)(20)$ is designed to measure verbal learning and memory using a multiple-trial list-learning task. The Doors Test (21) is a visual recognition memory test. Trail Making Test reflects the control of attention, visual exploration, speed and mental flexibility. The Verbal Fluency Test (22) evaluates the spontaneous production of words under restricted search conditions. 


\section{Clinical Staging}

In order to classify patients into 5 clinical stages, we used the three clinical indicators used by McGorry et al (2010) criteria for clinical staging (2) (see Supplementary Figure):

-The severity of symptoms, using the PANSS total score

- Recurrence or relapses based the number of lifetime psychotic episodes

- Global functioning, using the GAF score

These 5 clinical stages ranged from favorable functioning and no symptoms (stage II) to unremitted illness and poor functioning (stage IV). According to the characteristics of our cohort, which included only patients after at least a first psychotic episode, stages 0 , Ia and Ib were not part of the cohort, given that they describe patients at risk of psychosis.

\section{Statistical Analysis}

Sociodemographics, clinical data, and treatments are presented as the mean $+/$ - the standard deviation (SD) for continuous variables and frequency distribution for categorical variables.

We performed unsupervised machine learning algorithm using clustering analysis based on kmeans estimation. In order to check the stability of our cluster, we generated a simple random sample without replacement of 500 patients from the original dataset and rerun the cluster analysis (not shown). Analyses of (co)variance were then performed to compare each group in regard to socio-demographics factors, clinical characteristics, co-morbidities, therapeutics and cognitive parameters. Continuous variables were analyzed with the Student $t$-test for normally distributed data and the Mann-Whitney test in case of non-normal distribution. Neuropsychological test were adjusted for age, gender and education level. In order to explore the evolution of each stage, in the subsample of 297 patients followed-up for one year, we calculated the proportion of individuals that started and ended the study at the same stage and the proportion of patients that evolved from one stage to another. For this exploratory analysis, we grouped together stage IIa and stage IIb as well as stages IIIa and IIIb. 
Comparisons between scores at baseline and scores at one-year using the Wilcoxon signedrank test were also performed. Analyses were conducted using SAS (release 9.3; SAS Statistical Institute, Cary, NC). All statistical tests were two-tailed, with the $\alpha$ level set at 0.05 . 


\section{Results}

\section{Characteristics of the sample}

770 stable SZ outpatients (mean age 32 years, $74 \%$ male) were included in the FACE-SZ cohort. Among the 770 patients assessed at the initial visit, 325 were evaluated at one-year. There were no significant differences between patients seen at the beginning of the study and those lost during follow-up in regard to our variables of interest (severity of the disease, level of functioning, number of lifetime episode, cognitive abilities) (all $\mathrm{p}>0.05$, data not shown). Of the 325 patients with two assessments at one year interval, 297 had sufficient information available to allow clinical stage assessment.

\section{Stages: clinical characteristics}

The unsupervised machine learning algorithm classified patients into 5 clinically meaningful stages, defined as stages IIa ( $\mathrm{n}=89)$, IIb $(\mathrm{n}=272)$, IIIa $(\mathrm{n}=241)$, IIIb $(\mathrm{n}=112)$ and IV $(\mathrm{n}=56)$, ranging from high functioning and no symptoms to current severe symptomatology and poor functioning (Figure 1). The Grade IIa corresponds to patients with clinical full remission (mean PANSS score $43.9 \pm 6.8$ / asymptomatic) and good functioning (mean GAF score 69.3 \pm 9.7). In Grade IIb, patients had mild symptomatology (mean PANSS score $59.4 \pm 6.5$ ) and mildly impaired functioning (mean GAF score $52.8 \pm 7.7$ ) compared to patients in Grade IIa. The Grade IIIa included patients with incomplete remission (mean PANSS score $76.1 \pm 6.7$ ) and a moderate level of functioning (mean GAF score $46.0 \pm 8.0$ ). Grade IIIb corresponds to severely ill patients (PANSS score $91.4 \pm 6.3$ ) with a correspondingly severe impairment in functioning (mean GAF score $36.7 \pm 8.2$ ). The last grade (Grade IV) represents the most advanced stage of the illness, corresponding to patients who have extremely high levels of symptomatology (PANSS score 113.5 \pm 11.6 ) and highly impaired global functioning (mean GAF score 31.2 \pm 8.4 ). 
Table 1 shows comparison of the 5 clinical stages according to socio-demographics factors, clinical characteristics, co-morbidities and treatments. Higher stage grade is associated with lower education level, higher depressive and manic symptoms, and lower self-rated quality of life. More precisely, $42.6 \%$ of patients in stage IV had depressive symptoms satisfying criteria for a major depressive episode (mean Calgary score above 6), compared to $3.4 \%$ in stage IIa and $19.6 \%$ in stage $\mathrm{IIb}(\mathrm{p}<0.0001$ and $\mathrm{p}=0.0006$ respectively). Notably, comorbidities such as current comorbid anxiety disorder and daily tobacco smoking, as well as cannabis or alcohol use disorders or BMI did not differ significantly among stages. Analysis related to medication factors showed a linear relationship between stages and adherence to medication. More advanced stage was associated with lower adherence to medication, and a higher percentage of first-generation antipsychotic medications $(51.2 \%$ in stage IV, versus $20.8 \%$ in stage II, $\mathrm{p}=0.0002$ ). More advanced stage was also associated with a higher number of antipsychotic and psychotropic medications, higher chlorpromazine equivalent doses, and a higher rate of extrapyramidal symptoms, namely drug-induced Parkinsonism and akathisia. We did not observe significant associations between CRP, whole blood count, lipid profile and clinical stage (data not shown).

Cognitive performances of each of the clinical stage is shown in Table 2. After adjustment for age, gender and education level, more advanced stage stage was associated with a lower level of cognitive performance, especially current and premorbid intellectual functioning, working memory, executive function, learning abilities and semantic memory.

\section{Course of each subgroup at one year: exploratory analysis}

In the subsample of 297 patients followed at one year, we explored principal outcome profiles of each stage at one year (Table 3). Globally, we observed that patients in stage II were stable over one year of follow-up, whereas patients in other stages significantly improved in terms 
of symptomatology, functioning, depressive symptoms, and adherence to medication as well as quality of life. Patient in the more advanced stages had consistently poorer prospective outcomes.

We then explored the evolution of each stage at one year in relation to the initial baseline stage (Figure 2). The majority of the patients were in stage II and stage III at baseline and after one year remained in the same stage. Approximately $62 \%$ remained stable and $38 \%$ of patients changed stages over time. Among those who changed, $43 \%$ improved to a better grade, whilst deterioration was observed in 57\% (64/113 patients). More specifically, among patients in stage II $(n=137), 67 \%$ remained stable at one year, $30 \%$ deteriorated to stage III and $3 \%(n=4)$ deteriorated to stage IV. Patients in intermediate stage (stage III, $\mathrm{n}=146$ ) remained globally stable (58\%) or improved to stage II at one year $(28 \%)$. Of patients in stage IV at inclusion $(n=14), 57 \%$ remained stable, indicating that less than half of stage IV patients improved at one year, with none achieving full remission and a high level of functioning. 


\section{Discussion}

In a large French prospective cohort of stable SZ outpatients, using unsupervised machine learning, we were able to classify patients in 5 stages from II to $\mathrm{V}$. We showed that the model proposed by McGorry et al. in 2010 (2) can be improved by sub-dividing the intermediate stages (II and III) and by adding clinical elements such as mood symptoms and assessment of cognitive handicap. It is important to note that this is based on data-driven cluster analysis, which assumes no a priori specific hypotheses or thresholds. Our findings also showed that, compared to other stages, patients in stage IV were very depressed, more frequently received first-generation antipsychotics and had more severe cognitive impairment. Follow-up at one year indicated that the majority of patients remained stable in all stages. Less than half of baseline stage IV patients improved at one year. Importantly, this data suggests a reverse gear to clinical staging, traditionally thought as uniformly progressive, with improvement not only possible but common in the earlier but not the later stages.

Despite the widespread utilization of clinical staging across many branches of medicine, few studies have applied clinical staging models in individuals with schizophrenia. Tedja et al., in a cohort of 649 patients diagnosed with schizophrenia followed-up for 3 years, assigned patients to a clinical stage according to methods described by McGorry in 2010. The authors concluded the intermediate stages could be improved, although suggesting the highest and lowest symptomatic stages would be usefully differentiated by classical psychosis symptomatology, as well as social and neurocognitive functioning (9). These authors note that the study was limited by its retrospective design and the absence of treatment data in the staging model. The current investigation builds on these previous studies, including by better defining group stages by the utilization of cluster analysis, which is based on predefined clinical and functional criteria. The results of the present study support the suggestion that the intermediate stages could be improved, with the data indicating the validity of stage II and III 
subgroups.

The presented data show the most severe stages to be associated with greater cognitive impairment, including intellectual functioning, working memory, executive function, learning abilities and semantic memory. This, in conjunction with the data showing that $30 \%$ of stage II patients shift to stage III at follow up, may be parsimonious with concepts of neuroprogression in patients with schizophrenia (23). The data also showed that lower premorbid intellectual functioning and a lower education level was associated with the most severe stages, indicating that early development may contribute to the severity of symptomatology in adults diagnosed with schizophrenia, and may contribute to accelerated neuroprogression.

The role of mood symptomatology in schizophrenia has long been noted. In the current study, depression and excitement were evaluated using standardized tools (CDRS and PANSS depressive factor for depressive symptoms and YMRS and PANSS excitement factor for manic symptoms). Notably, depression was strongly over-represented in stage IV (and my drive the later stages), which is consistent with previous results showing heightened levels of depression in SZ patients with greater symptomatology $(9,24-27)$. This is also consistent with the only study exploring clinical stages in individuals with schizophrenia, which showed depressive symptoms were associated with the transition to more chronic stages (9). Mood symptoms in SZ are an important aspect of symptomatology (28). The DSM-5, in section III, recommends a dimensional evaluation of positive, negative, and cognitive as well as depressive and manic symptoms in SZ patients, highlighting the clinical relevance of alterations in mood as well as classic symptomatology (29). This could suggest that the recognition and treatment of the pathophysiological underpinnings of depression in SZ diagnosed patients may modulate the neuroprogressive course of SZ, and therefore shifts to 
more severe clinical stages. It also highlights the importance of aggressively treating mood in people with schizophrenia. Future longitudinal studies are required to investigate this.

Given that clozapine is recommended for resistant SZ, it would have been expected that most patients at stage IV would have been administered clozapine. However, this was not the case, with patients at this stage being more frequently prescribed first-generation antipsychotics. This is surprising, given the ongoing debate regarding the long-term brain toxicity of these drugs (30). This also suggests the possibly iatrogenic role of guideline non-concordant therapy in driving illness progression. Patients in the more severe stages may also be overprescribed medications, as shown by higher rates of chlorpromazine equivalents, antipsychotic polytherapy and extrapyramidal side effects. As the present study has a crosssectional design, a causal relationship cannot be inferred. However, it may reasonably be suggested that the following interventions should be systematically used and evaluated in stage III and stage IV patients: clozapine administration over 6 months (31), benzodiazepine and hypnotic withdrawal, antipsychotic monotherapy, using antipsychotics plasma level monitoring to adjust dosage and to reduce extrapyramidal symptoms and aggressive treatment of depression (32,33). It also suggests a clinical pathway why antidepressants may be useful for schizophrenia(34). Another major finding of the current study was rates of shift to different stages at one year follow-up. We showed that the majority of patients remained stable in all stages. Of those that shifted to different stages, $40 \%$ moved to a lower stage and $60 \%$ to a higher stage. During this one-year follow-up, $30 \%$ of stage II patients moved to a more severe stage, although none transitioned to stage IV. A non-negligible proportion of patients in stage IV improved, although none showed improvement to stage IIa. Clearly, a one-year follow up is a relatively short timespan to evaluate the results of an intervention or illness course. However, the results do suggest that at least some patients in all baseline stages may improve $(35,36)$. The improvement of the patients in the stage IV at one year-follow-up 
may be due to multiple factors associated with the Expert Center intervention including pharmacological intervention (treatments modifications including treating major depression by antidepressants, antipsychotic switch to clozapine, benzodiazepine withdrawal, withdrawing anticholinergics and first-generation antipsychotics), psychotherapy (including Cognitive Behavioral Therapy, Cognitive Remediation Therapy), lifestyle (diet, physical activity, sleep, addictions). This intervention has been comprehensively described elsewhere (37). Future studies should determine more precisely which intervention has the greatest impact on staging improvement. Due to the sample size, it was not possible to identify the factors associated with this improvement, which is a limitation of the present study. However, a clear picture of appropriate clinical stages in SZ should help to clarify the relevant biological underpinnings, which future medication should target, and which will eventually better define clinical stages and prevent the consequences of neuroprogression in this devastating disorder.

Perspectives. Future studies are warranted to investigate the validity of our proposed clinical staging. External biological validators may also support the staging construct. For this project, only CRP, cell blood count, and lipid profiles were available. In line with the inflammatory hypothesis of psychotic disorders, we explored the link between C-reactive protein (CRP) and the defined clinical stages, but no association was observed (data not shown). Previous data has indicated that elevated CRP levels is predominantly correlated with illness severity, mostly during the recrudescent phase (38). The lack of any association with CRP levels in the present investigation may be seen as fitting the mixed results associated with CRP levels in SZ studies, at least in part as a consequence of methodological issues. Future investigations of clinical staging in SZ should explore the relevance of different inflammatory, endocrine, neurotransmitter blood biomarkers as well as brain morphometric parameters (39). By tying neuroprogression to pathophysiological processes, such studies should better define relevant 
pharmaceutical targets. Applying treatment guideline recommendations for resistant schizophrenia would appear to be a first-step intervention for higher stage patients, and requires further evaluation.

Limits. A high rate (50\%) of participants were lost during follow-up. However, there were no significant differences at baseline between participants who completed the study and those who did not attend the follow-up. Longitudinal clinical studies on psychiatric patients are more complicated than those on the general population, especially for SZ patients. Moreover, the small sample size in each stage at one year follow-up did not allow the exploration of associated factors. Consequently, these results should be treated as preliminary. The FACESZ cohort will allow for further follow-up on these patients at 2 and 3 years, which should make their interpretation more definitive (40). Nevertheless, our results do provide an initial overview of the potential use of this model.

Strengths. Contrary to the previous studies, stage definition in the present investigation utilized cluster analysis. Compared to previous studies, the present investigation was not a post-hoc analysis, but has a prospective design, which reinforces the strength of present results. A wide array of clinical and neuropsychological variables are reported, including treatments, unlike in previous studies. This strengthens the validity of the stages derived herein. The present results also highlight the potential utility of treatment in the more severely symptomatic stages, which has long been challenging for clinical psychiatry.

In conclusion, in outpatients with schizophrenia, clinical staging model proposed by McGorry et al, may be improved by sub-dividing stages II and III including total remission and moderate to good functioning. The current study has highlighted the role of mood dysregulation and cognitive impairment in the more symptomatic stages of SZ. In terms of therapeutic strategies, treating depression, reducing the number of psychotropic medication, improving adherence to treatment, and prescribing cognitive remediation early have to 
emphasized to prevent chronicity. It will be important for future studies to look at longer term follow-up and to investigate wider ranges of pathophysiological data, which should help in determining a better prognosis, as well as more refined pharmaceutical treatment targets.

\section{Conflict of interest}

No conflicts to disclose

\section{Acknowledgments}

We thank the FondaMental Foundation (www-fondation-fondamental.org) which is a nonprofit foundation supporting research in psychiatry in France and coordinating the infrastructure of Bipolar Expert Centers. We express all our thanks to the patients who have accepted to be included in the present study. We thank the team of FondaMental foundation, Hakim Laouamri and his team (Seif Ben Salem, Karmène Souyris,Victor Barteau and Mohamed Laaidi) for the development of the FACE-SZ computer interface, data management, quality control and regulatory aspects..

\section{Funding sources}

This work was funded by AP-HP (Assistance Publique des Hôpitaux de Paris), Fondation FondaMental (RTRS Santé Mentale), by the Investissements d'Avenir program managed by the ANR under reference ANR-11-IDEX-0004-02 and ANR-10-COHO-10-01, and by INSERM (Institut National de la Santé et de la Recherche Médicale). This funding source had no role in the study design, data collection, analysis, preparation of the manuscript, or decision to submit the manuscript for publication. O Godin had full access to all the data in the study and takes responsibility for the integrity of the data and the accuracy of the data analysis. All authors have approved the manuscript and have nothing to disclose. 
Authors' contributions

Conception and design: ML, PML

Inclusion and clinical data collection: All authors except OG and DC

Analysis of data: OG.

Interpretation of data: OG, GF, ML, DC, PML.

Drafting and writing of the manuscript: all of the authors. 
Reference

1. FAVA GA, KELLNER R. Staging: a neglected dimension in psychiatric classification. Acta Psychiatr Scand 1993;87:225-230.

2. McGorry PD, Nelson B, Goldstone S, Yung AR. Clinical staging: a heuristic and practical strategy for new research and better health and social outcomes for psychotic and related mood disorders. Can J Psychiatry 2010;55:486-497.

3. SCOTT J, LEBOYER M, HickiE I et al. Clinical staging in psychiatry: a cross-cutting model of diagnosis with heuristic and practical value. Br J Psychiatry 2013;202:243-245.

4. Rosa AR, Magalhães PVS, CZePIElewski L et al. Clinical staging in bipolar disorder: focus on cognition and functioning. J Clin Psychiatry 2014;75:e450-456.

5. Magalhães PV, Dodd S, Nierenberg AA, Berk M. Cumulative morbidity and prognostic staging of illness in the Systematic Treatment Enhancement Program for Bipolar Disorder (STEP-BD). Aust N Z J Psychiatry 2012;46:1058-1067.

6. Grande I, Magalhães PV, Chendo I et al. Staging bipolar disorder: clinical, biochemical, and functional correlates. Acta Psychiatr Scand 2014;129:437-444.

7. Carrión RE, Correll CU, Auther AM, Cornblatt BA. A Severity-Based Clinical Staging Model for the Psychosis Prodrome: Longitudinal Findings From the New York Recognition and Prevention Program. Schizophr Bull 2017;43:64-74.

8. ORTIZ BB, EDEN FDM, DE SOUZA ASR et al. New evidence in support of staging approaches in schizophrenia: Differences in clinical profiles between first episode, early stage, and late stage. Compr Psychiatry 2017;73:93-96.

9. Tedja A, Velthorst E, van Tricht M, de HaAn L, GROUP. Preliminary validation of a clinical staging model in schizophrenia and related disorders. Clin Schizophr Relat Psychoses Published Online First: 4 August 2017. doi:10.3371/CSRP.ATEV.071317

10. GUELFI JD. [The PANSS (Positive And Negative Symptom) Scale]. Encephale 1997;23 Spec No 2:35-38.

11. Wallwork RS, Fortgang R, Hashimoto R, Weinberger DR, Dickinson D.

Searching for a consensus five-factor model of the Positive and Negative Syndrome Scale for schizophrenia. Schizophr Res 2012;137:246-250.

12. Bernard D, Lançon C, Auquier P, Reine G, Addington D. Calgary Depression Scale for Schizophrenia: a study of the validity of a French-language version in a population of schizophrenic patients. Acta Psychiatr Scand 1998;97:36-41.

13. Young RC, Biggs JT, Ziegler VE, Meyer DA. A rating scale for mania: reliability, validity and sensitivity. Br J Psychiatry 1978;133:429-435. 
14. Misdrahi D, Verdoux H, LlorCA P-M, BAYLÉ F-J. [Therapeutic adherence and schizophrenia: the interest of the validation of the French translation of Medication Adherence Rating Scale (MARS)]. Encephale 2004;30:409-410.

15. Leucht S, Samara M, Heres S, Patel MX, Woods SW, Davis JM. Dose equivalents for second-generation antipsychotics: the minimum effective dose method. Schizophr Bull 2014;40:314-326.

16. BARNES TR. A rating scale for drug-induced akathisia. Br J Psychiatry 1989;154:672676.

17. Lejoyeux M, Gorwood P, Stalla-Bourdillon A, AdÈs J. [Translation and application of the Simpson and Angus Scale of Extrapyramidal Symptoms]. Encephale 1993;19:17-21.

18. Nelson HE, O'CONNELl A. Dementia: the estimation of premorbid intelligence levels using the New Adult Reading Test. Cortex 1978;14:234-244.

19. WECHSLER D. Echelle d'intelligence de Wechsler pour adultes:WAIS-III. Les Editions du Centre de Psychologie Appliquée. 2008

20. Delis D, Kramer, JH, Kaplan E, OBer BA. California Verbal Learning Test.The Psychological Corporation. Harcourt Assessment Company. San Antonio, TX, 2000

21. Baddeley AD, Emslie H, Nimmo-Smith I. Doors and People: A Test of Visual and Verbal Recall and Recognition. Thames Valley Test Company, 1994

22. Cardebat D, Doyon B, Puel M, Goulet P, Joanette Y. Evocation lexicale formelle et sémantique chez des sujets normaux. Performances et dynamiques de production en fonction du sexe, de l'âge et du niveau d'étude. Acta Neurologica Belgica. 1990;:90(4) 207-217.

23. Davis J, Moylan S, Harvey BH, Maes M, Berk M. Neuroprogression in schizophrenia: Pathways underpinning clinical staging and therapeutic corollaries. Aust N Z J Psychiatry 2014;48:512-529.

24. ANDRIANARISOA M, BOYER L, GODIN O et al. Childhood trauma, depression and negative symptoms are independently associated with impaired quality of life in schizophrenia. Results from the national FACE-SZ cohort. Schizophr Res Published Online First: 18 January 2017. doi:10.1016/j.schres.2016.12.021

25. Godin O, LEBOYER M, SCHÜRHOFF F et al. Predictors of rapid high weight gain in schizophrenia: Longitudinal analysis of the French FACE-SZ cohort. J Psychiatr Res 2017;94:62-69.

26. KRYNICKI CR, UPTHEGROVE R, DEAKIN JFW, BARNES TRE. The relationship between negative symptoms and depression in schizophrenia: a systematic review. Acta Psychiatr Scand 2018;137:380-390. 
27. REY R, D'AMATO T, BOYER L et al. Nicotine dependence is associated with depression and childhood trauma in smokers with schizophrenia: results from the FACE-SZ dataset. Eur Arch Psychiatry Clin Neurosci Published Online First: 7 April 2017.

28. VAN Os J, KAPUR S. Schizophrenia. Lancet 2009;374:635-645.

29. AmEriCAn PSYCHIATRIC Association. Diagnostic and Statistical Manual of Mental Disorders , Fifth Edition. Arlington: American Psychiatric Publishing. 2013

30. Solmi M, Murru A, Pacchiarotti I et al. Safety, tolerability, and risks associated with first- and second-generation antipsychotics: a state-of-the-art clinical review. Ther Clin Risk Manag 2017;13:757-777.

31. NATIONAL InSTITUTE FOR CLINICAL EXCELLENCE (NICE). Technology appraisal Guidance No. 43. Guidance on the use of newer (atypical) antipsychotic drugs for the treatment of schizophrenia. London, 2002

32. BERNA F, MisDRAHI D, BOYER L et al. Akathisia: prevalence and risk factors in a community-dwelling sample of patients with schizophrenia. Results from the FACE-SZ dataset. Schizophr Res 2015;169:255-261.

33. FOND G, BERNA F, BOYER L et al. Benzodiazepine long-term administration is associated with impaired attention/working memory in schizophrenia: results from the national multicentre FACE-SZ data set. Eur Arch Psychiatry Clin Neurosci 2018;268:17-26.

34. Singh SP, Singh V, KAR N, CHAN K. Efficacy of antidepressants in treating the negative symptoms of chronic schizophrenia: meta-analysis. Br J Psychiatry 2010;197:174179.

35. Berna F, GÖRITZ AS, Llorca P-M, Vidailhet P, Fond G, Moritz S. Would I take antipsychotics, if I had psychotic symptoms? Examining determinants of the decision to take antipsychotics. Prog Neuropsychopharmacol Biol Psychiatry 2017;77:155-163.

36. Samalin L, Guillaume S, Auclair C, LlorCa P-M. Adherence to guidelines by French psychiatrists in their real world of clinical practice. J Nerv Ment Dis 2011;199:239243.

37. SCHÜRHOFF F, FOND G, BERnA F et al. [The 10-year findings from the FondaMental Academic Center of Expertise for Schizophrenia (FACE-SZ): Review and recommendations for clinical practice]. Encephale Published Online First: 13 October 2018.

38. Orsolini L, SARChione F, Vellante F et al. Protein-C Reactive as Biomarker Predictor of Schizophrenia Phases of Illness? A Systematic Review. Curr Neuropharmacol 2018;16:583-606.

39. DwYer DB, CABral C, KAMBEITZ-Ilankovic L et al. Brain Subtyping Enhances The Neuroanatomical Discrimination of Schizophrenia. Schizophr Bull Published Online First: 26 February 2018. 
40. SCHÜRHOFF F, FOND G, BERNA F et al. A National network of schizophrenia expert centres: An innovative tool to bridge the research-practice gap. Eur Psychiatry 2015;30:728735. 
Table 1: Association between socio-demographics factors, clinical characteristics and co-morbidities of each stage

\begin{tabular}{|c|c|c|c|c|c|c|}
\hline & $\begin{array}{c}\text { Stage IIa } \\
n=89\end{array}$ & 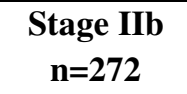 & $\begin{array}{c}\text { Stage IIIa } \\
n=241\end{array}$ & $\begin{array}{c}\text { Stage IIIb } \\
n=112\end{array}$ & $\begin{array}{c}\text { Stage IV } \\
n=56\end{array}$ & P value* \\
\hline \multicolumn{7}{|l|}{ Socio demographic characteristics } \\
\hline Age mean (sd) (years) & $32.5(7.7)$ & $31.8(9.5)$ & $32.5(10.5)$ & $33.0(11.0)$ & $32.7(9.7)$ & 0.81 \\
\hline \multicolumn{7}{|l|}{ Gender } \\
\hline Men & $60(67.4)$ & $208(76.5)$ & $179(74.3)$ & $79(70.5)$ & $44(78.6)$ & 0.38 \\
\hline Women & $29(32.6)$ & $64(23.5)$ & $62(25.7)$ & $33(29.5)$ & $12(21.4)$ & \\
\hline Education level (years), mean (sd) & $13.1(2.8)$ & $12.7(2.8)$ & $12.1(2.7)$ & $11.0(2.4)$ & $11.4(2.9)$ & $<0.0001$ \\
\hline \multicolumn{7}{|l|}{ Illness characteristics } \\
\hline Age of SZ onset (years), mean (sd) & $21.7(5.7)$ & $22.4(6.9)$ & $21.3(6.1)$ & $20.6(6.7)$ & $20.5(6.3)$ & 0.10 \\
\hline $\mathrm{DUP}^{\dagger}$, mean (sd) & $1.6(3.2)$ & $1.2(2.9)$ & $1.7(3.5)$ & $1.2(2.6)$ & $1.2(2.8)$ & 0.39 \\
\hline \multicolumn{7}{|l|}{ Panss 5 factors } \\
\hline Positive & 6.0 (2.2) & $7.5(3.0)$ & $9.9(4.0)$ & 12.1 (3.9) & $16.1(4.6)$ & $<0.0001$ \\
\hline Negative & $10.0(3.6)$ & $14.8(4.5)$ & $19.0(5.2)$ & $23.1(5.5)$ & $25.2(6.4)$ & $<0.0001$ \\
\hline Cognitive & $5.0(1.8)$ & $6.8(2.5)$ & $8.8(2.7)$ & $11.0(3.0)$ & $13.5(3.7)$ & $<0.0001$ \\
\hline Excitement & $4.5(1.1)$ & $4.8(1.4)$ & $6.0(2.2)$ & $6.7(2.7)$ & $9.7(3.6)$ & $<0.0001$ \\
\hline Depressive & $4.8(2.0)$ & $6.7(2.7)$ & $7.9(3.1)$ & $8.0(3.4)$ & $10.2(3.9)$ & $<0.0001$ \\
\hline Depressive symptoms ${ }^{\ddagger}$, mean sd) & $1.5(2.2)$ & $3.4(3.6)$ & $4.5(4.4)$ & $5.4(4.8)$ & $6.5(6.0)$ & $<0.0001$ \\
\hline Manic Symptoms ${ }^{\S}$, mean (sd) & $1.6(3.4)$ & $1.8(3.2)$ & $2.9(4.7)$ & $2.7(3.5)$ & $3.7(5.1)$ & 0.0016 \\
\hline \multicolumn{7}{|l|}{ Comordidities } \\
\hline BMI, mean (sd) & $25.6(4.8)$ & $26.0(4.9)$ & $26.3(5.1)$ & $26.2(5.2)$ & $26.6(6.1)$ & 0.85 \\
\hline \multicolumn{7}{|l|}{ Current anxiety disorder, $\mathrm{n}(\%)$} \\
\hline No & $53(63.1)$ & $152(57.8)$ & $133(58.6)$ & $64(63.4)$ & $34(69.4)$ & 0.51 \\
\hline Yes & $31(36.9)$ & $111(42.2)$ & $94(41.4)$ & $37(36.6)$ & $15(30.6)$ & \\
\hline \multicolumn{7}{|l|}{ Current daily tobacco smoking, $\mathrm{n}(\%)$} \\
\hline No & $35(43.2)$ & $127(49.4)$ & $105(45.3)$ & $44(40.7)$ & $20(40.0)$ & 0.50 \\
\hline Yes & $46(56.8)$ & $130(5.6)$ & $127(54.7)$ & $64(59.3)$ & $30(60.0)$ & \\
\hline \multicolumn{7}{|l|}{ Lifetime cannabis use disorder, $\mathrm{n}(\%)$} \\
\hline No & $26(48.2)$ & $121(62.0)$ & $95(58.3)$ & $39(56.5)$ & $16(45.7)$ & 0.23 \\
\hline Yes & $28(51.9)$ & $74(37.9)$ & $68(41.7)$ & $30(43.5)$ & $19(54.3)$ & \\
\hline \multicolumn{7}{|l|}{ Lifetime alcohol use disorder, $\mathrm{n}(\%)$} \\
\hline No & $42(77.8)$ & 149 (71.6) & $126(71.6)$ & $45(66.2)$ & $23(67.7)$ & 0.70 \\
\hline
\end{tabular}




\begin{tabular}{|c|c|c|c|c|c|c|}
\hline Yes & $12(22.2)$ & $59(28.4)$ & $50(28.4)$ & $23(33.8)$ & $11(32.4)$ & \\
\hline \multicolumn{7}{|l|}{ Current cannabis use } \\
\hline No & $45(88.2)$ & $173(93.0)$ & $132(85.7)$ & $53(82.8)$ & $29(90.6)$ & 0.13 \\
\hline Yes & $6(11.8)$ & $13(7.0)$ & $22(14.3)$ & $11(17.2)$ & $3(9.4)$ & \\
\hline \multicolumn{7}{|l|}{ Current alcohol use } \\
\hline No & $53(98.2)$ & $186(91.6)$ & $154(89.5)$ & $59(92.2)$ & $31(93.9)$ & 0.37 \\
\hline Yes & $1(1.8)$ & $17(8.4)$ & $18(10.5)$ & $5(7.8)$ & $2(6.1)$ & \\
\hline \multicolumn{7}{|l|}{ Treatment } \\
\hline Adherence to medication & $7.1(2.1)$ & $6.4(2.1)$ & $6.1(2.4)$ & $5.6(2.3)$ & $5.6(2.3)$ & $<0.0001$ \\
\hline First Generation Antipsychotic, n(\%) & $16(20.8)$ & $45(19.9)$ & $51(27.0)$ & $29(32.6)$ & $22(51.2)$ & 0.0002 \\
\hline Antidepressant, $\mathrm{n}(\%)$ & $16(20.8)$ & $75(33.2)$ & $66(34.9)$ & $30(33.7)$ & $11(25.6)$ & 0.18 \\
\hline Clozapine, $\mathrm{n}(\%)$ & $12(15.6)$ & $32(14.2)$ & $36(19.1)$ & $19(21.3)$ & $9(20.9)$ & 0.47 \\
\hline Number of antipsychotropic treatment & $1.2(0.6)$ & $1.3(0.6)$ & $1.3(0.6)$ & $1.3(0.6)$ & $1.6(0.7)$ & 0.02 \\
\hline Number of psychotropic medication & $2.0(1.1)$ & $2.3(1.3)$ & $2.5(1.4)$ & $2.7(1.5)$ & $2.9(1.5)$ & 0.0010 \\
\hline CPZeq mean $(\mathrm{sd})$ & $487.1(392.9)$ & $510.8(442.2)$ & $678.7(748.2)$ & $646.6(561.4)$ & $940.4(873.7)$ & 0.0001 \\
\hline Parkinsonism, n(\%) & $2(2.6)$ & $14(5.6)$ & $12(5.5)$ & $6(5.9)$ & $12(27.3)$ & $<0.0001$ \\
\hline Akathisia, $\mathrm{n}(\%)$ & $8(9.6)$ & $31(12.2)$ & $41(18.2)$ & $19(18.1)$ & $12(25.0)$ & 0.0538 \\
\hline
\end{tabular}

*Chi-square for categorical variables and t-test or Mann-Whitney test for continues variables

${ }^{\dagger}$ Duration of Untreated Psychosis.

\$Evaluated using Calgary Depression Rating Scale

${ }^{\S}$ Evaluated using the Young Mania Rating Scale 
Table 2: Association between cognitive parameters and clinical stages.

\begin{tabular}{|c|c|c|c|c|c|c|}
\hline & $\begin{array}{c}\text { Grade IIa } \\
n=89\end{array}$ & $\begin{array}{c}\text { Grade IIb } \\
\mathbf{n}=272\end{array}$ & $\begin{array}{c}\text { Grade IIIa } \\
\mathbf{n}=241\end{array}$ & $\begin{array}{c}\text { Grade IIIb } \\
\mathbf{n}=112\end{array}$ & $\begin{array}{c}\text { Grade IV } \\
n=56\end{array}$ & P value $^{*}$ \\
\hline \multicolumn{7}{|l|}{ Intellectual ability, mean (sd) } \\
\hline Premorbid IQ, mean (SD) & $25.6(5.4)$ & $24.7(5.7)$ & $24.1(6.0)$ & $22.0(6.4)^{*}$ & $21.9(6.6)^{*}$ & 0.0001 \\
\hline Full Scale IQ, mean (SD) & $93.5(13.7)^{*}$ & $86.4(14.9)^{*}$ & $82.4(14.8) *$ & $76.7(12.9)$ & $75.2(17.5)$ & $<0.0001$ \\
\hline \multicolumn{7}{|l|}{ Working memory, mean (sd) } \\
\hline Arithmetic (std score) & $8.8(3.0)$ & $7.9(3.2)$ & $7.0(3.0)$ & $6.0(2.4)$ & $5.8(2.9)$ & $<0.0001$ \\
\hline Digit span (std score) & $8.8(2.4)$ & $8.5(2.6)$ & $8.1(2.9)$ & $7.5(3.0)$ & $6.6(2.9)$ & $<0.0001$ \\
\hline Digit span forwards (std score) & $6.2(1.0)$ & $6.1(1.1)$ & $6.0(1.2)$ & $5.7(1.3)$ & $5.5(1.3)$ & 0.001 \\
\hline Digit span backwards (std score) & $4.4(1.1)$ & $4.4(1.1)$ & $4.2(1.1)$ & $4.1(1.3)$ & $3.6(1.0)$ & 0.02 \\
\hline \multicolumn{7}{|c|}{ Visual attention \& Speed of Processing, mean (sd) } \\
\hline Trail Making Test A (time) & $36.5(13.8)$ & $39.4(18.5)$ & $43.9(17.5)$ & $48.7(26.9)$ & $57.4(36.6)$ & $<0.0001$ \\
\hline Trail Making Test A (errors) & $0.27(0.5)$ & $0.18(0.5)$ & $0.20(0.5)$ & $0.24(0.6)$ & $0.07(0.3)$ & 0.14 \\
\hline Digit-Symbol Coding (std score) & $7.2(3.1)$ & $6.6(2.8)$ & $5.7(2.8)$ & $4.9(2.8)$ & $4.0(2.6)$ & $<0.0001$ \\
\hline Picture Completion (std score) & $9.1(2.9)$ & $7.6(3.2)$ & $7.3(3.3)$ & $6.5(3.3)$ & $6.7(3.6)$ & $<0.0001$ \\
\hline \multicolumn{7}{|l|}{ Executive functions, mean (sd) } \\
\hline Trail Making Test B (time) & $80.5(36.2)$ & $94.1(53.1)$ & $112.0(60.9)$ & $127.8(77.8)$ & $151.1(78.7)$ & $<0.0001$ \\
\hline Trail Making Test B (errors) & $0.39(0.8)$ & $0.62(1.2)$ & $0.86(1.7)$ & $1.00(1.6)$ & $1.18(1.8)$ & 0.0015 \\
\hline Trail Making Test B-A (time) & $44.0(29.7)$ & $54.9(41.0)$ & $68.2(51.4)$ & $81.3(68.9)$ & $96.7(59.3)$ & $<0.0001$ \\
\hline Set planning task, total correct & $893.2(26.5)$ & $839.8(14.8)$ & $793.4(16.2)$ & $716.01(23.7)$ & $691.9(42.3)$ & $<0.0001$ \\
\hline Set planning task, total errors & $5.9(0.6)$ & $6.8(0.3)$ & $7.7(0.4)$ & $9.1(0.5)$ & $9.8(1.0)$ & $<0.0001$ \\
\hline Similarities (std score) & $10.5(2.9)$ & $9.4(3.2)$ & $8.8(2.9)$ & $8.0(3.5)$ & $7.1(3.7)$ & $<0.0001$ \\
\hline Matrix reasoning (std score) & $9.7(2.9)$ & $8.4(3.3)$ & $7.7(3.3)$ & $6.9(3.2)$ & $6.9(3.3)$ & $<0.0001$ \\
\hline \multicolumn{7}{|c|}{ Learning abilities, episodic and semantic memory, mean $(s d)$} \\
\hline $\mathrm{CVLT}^{\dagger}$ recognition & $14.7(1.6)$ & $14.7(1.6)$ & $14.4(3.4)$ & $13.8(2.3)$ & $14.2(4.3)$ & 0.10 \\
\hline CVLT short delay free recall & $10.2(3.5)$ & $9.7(3.2)$ & $9.2(3.4)$ & $8.2(3.8)$ & $7.8(4.0)$ & 0.01 \\
\hline CVLT short delay cued recall & $11.0(3.1)$ & $10.4(2.8)$ & $9.7(3.3)$ & $8.9(3.6)$ & $8.5(3.5)$ & 0.002 \\
\hline CVLT long delay free recall & $10.7(3.2)$ & $10.3(3.2)$ & $9.5(3.5)$ & $8.5(3.8)$ & $8.1(3.7)$ & 0.0009 \\
\hline CVLT long delay cued recall & $11.0(3.2)$ & $10.4(3.0)$ & $9.7(3.5)$ & $8.9(3.7)$ & $8.5(3.5)$ & 0.003 \\
\hline Doors Test (A\&B) & $16.8(3.6)$ & $15.8(3.9)$ & $14.7(4.4)$ & $14.4(5.4)$ & $13.9(3.1)$ & 0.008 \\
\hline Information (std score) & $9.7(3.4)$ & $9.5(3.0)$ & $9.0(3.1)$ & $7.6(2.7)$ & $8.4(4.1)$ & $\mathbf{0 . 0 3}$ \\
\hline
\end{tabular}

\footnotetext{
${ }^{*}$ Analysis of covariance adjusted for age, gender and education level
}

${ }^{\dagger}$ California Verbal learning Test 
Table 3: Longitudinal outcomes at one year follow-up according to baseline stages

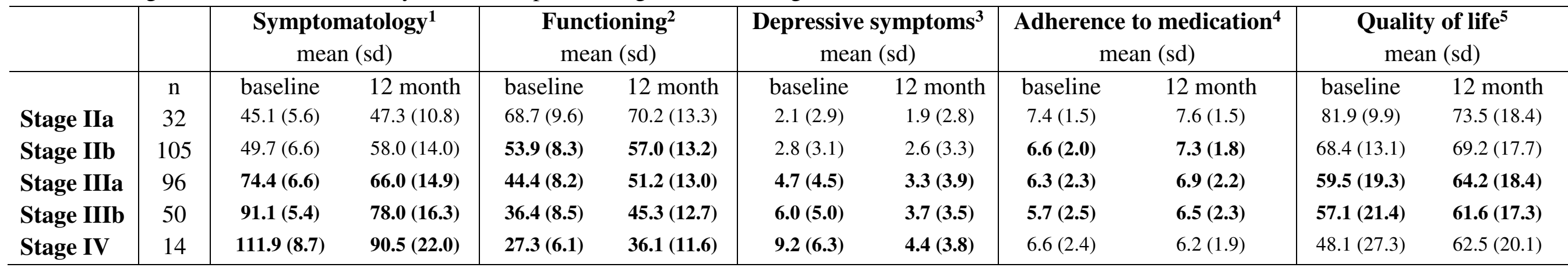

\footnotetext{
${ }^{1}$ Evaluated with Positive and Negative Syndrome Score (PANSS) total score

${ }^{2}$ Evaluated using the Global Assessment of Functioning (GAF) score

${ }^{3}$ Evaluated using the Calgary Depression Rating Scale

${ }^{4}$ Evaluated using the Medication Adherence Rating Scale

${ }^{5}$ Evaluated using the Medication Adherence Rating Scale

Comparisons in Bold are significant
} 
Figure 1: Classification of stabilized outpatients into 5 clinical stages

* Stabilized outpatients were classified into 5 clinical stages based on current illness severity, defined with the Positive and Negative Syndrome Score (PANSS) total score, the number of lifetime psychotic episodes and global functioning, using the Global Assessment of Functioning (GAF) score, ranging from favorable functioning and no symptoms (stage II) to unremitted illness and poor functioning (stage IV), according to the McGorry et al criteria.

Grade IIA corresponds to patients with no symptoms and good functioning. In Grade IIB, patients were paucisymptomatic and had a mildly impaired functioning compared to patients in Grade IIA. Grade IIIA corresponds to patients with incomplete remission and a moderate level of functioning. Grade IIIB corresponds to markedly ill patients, with poor functioning. The last grade (Grade IV) represents an advanced stage of the illness, corresponding to patients who are severely ill, with high severe impairment in global functioning. 
Figure 2: Evolution of clinical stages during one year follow-up according to initial stages at baseline

* At baseline

$\dagger$ At one-year follow-up 


\section{Proposed staging model for psychotic disorders}

\section{Stage Definition of stage}

0 Increased risk of psychotic or severe mood disorder; no symptoms currently

Ia Mild or nonspecific symptoms, including mild neurocognitive deficits of psychosis or severe mood disorder; mild functional change or decline

Ib Ultra-high risk: moderate but subthreshold symptoms, with moderate neurocognitive changes and functional decline to caseness (GAF 70)

II $\quad$ First episode of psychotic

Full threshold disorder with moderate-to severe symptoms, neurocognitive deficits and functional decline (GAF 30-50)

IIIa Incomplete remission from first episode of care; (could be linked or fasttracked to Stage IV)

IIIb Recurrence or relapse of psychotic which stabilizes with treatment at a level of GAF $\leq 30$, or with residual symptoms, or neurocognition below the best level achieved following remission from the first episode

IIIc Multiple relapses, when worsening in clinical extent and impact of illness is objectively present

IV Severe, persistent, or unremitting illness as judged on symptoms, neurocognition, and disability criteria

GAF : Global Assessment Functioning 
Figure 1: Clinical staging

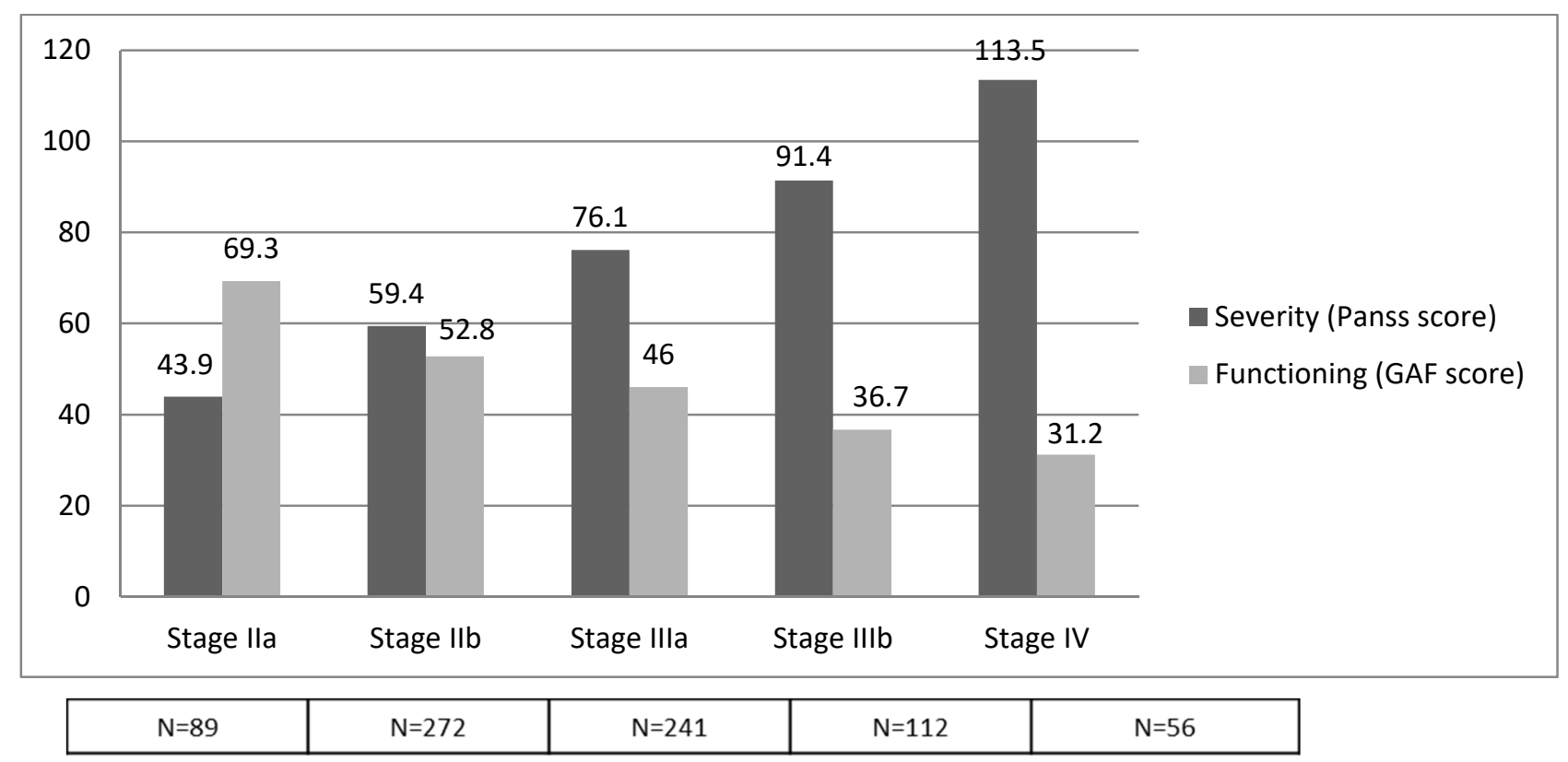

* Stabilized outpatients were classified into 5 clinical stages based on current illness severity, defined with the Positive and Negative Syndrome Score (PANSS) total score, the number of lifetime psychotic episodes and global functioning, using the Global Assessment of Functioning (GAF) score, ranging from favorable functioning and no symptoms (stage II) to unremitted illness and poor functioning (stage IV), according to the McGorry et al criteria (2010).

Grade IIA corresponds to patients with no symptoms and good functioning. In Grade IIB, patients were paucisymptomatic and had a mildly impaired functioning compared to patients in Grade IIA. Grade IIIA corresponds to patients with incomplete remission and a moderate level of functioning. Grade IIIB corresponds to markedly ill patients, with poor functioning. The last grade (Grade IV) represents an advanced stage of the illness, corresponding to patients who are severely ill, with high severe impairment in global functioning. 
Figure 1: Evolution of clinical stages during one year follow-up according to initial stages at baseline

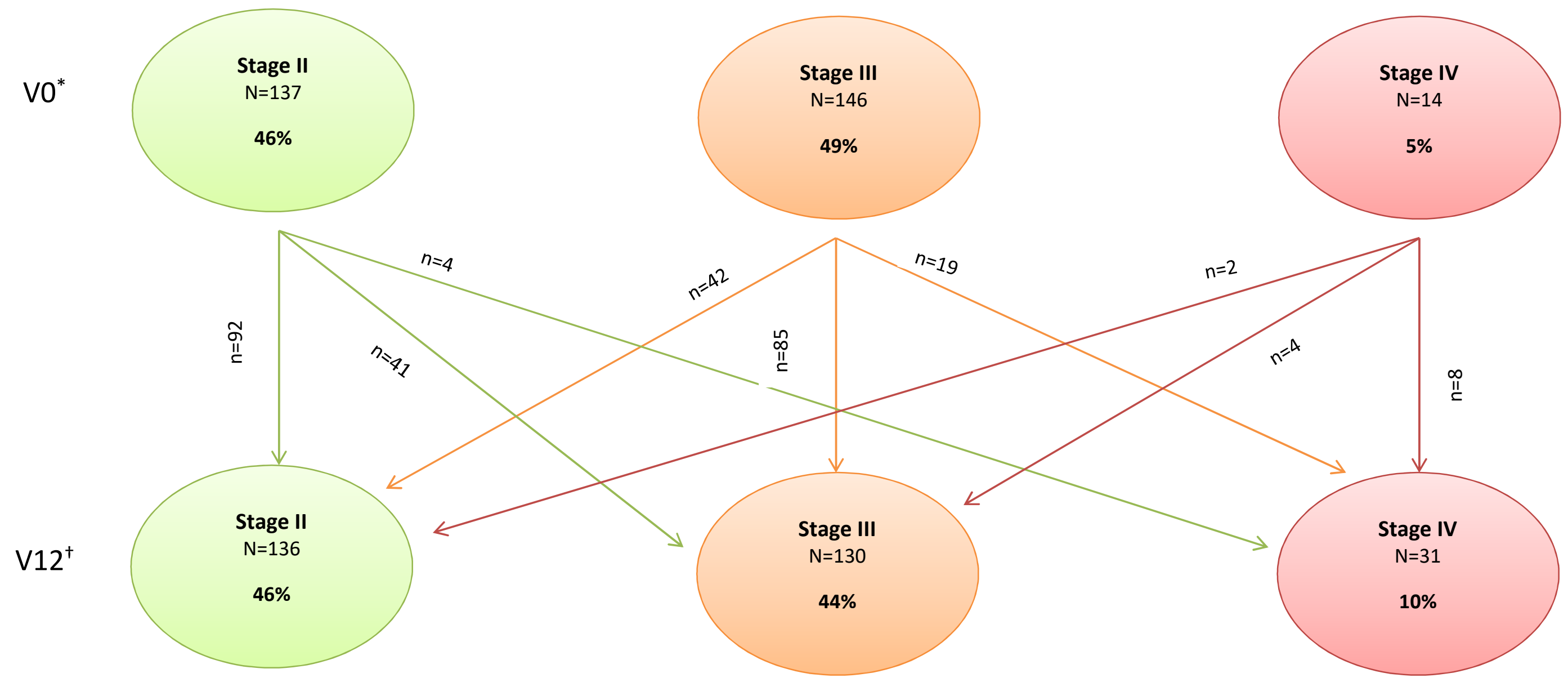

* At baseline

$\dagger$ At one-year follow-up 